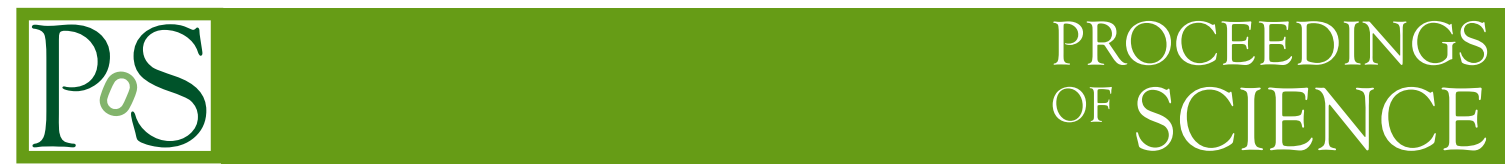

\title{
CTA constraints on AGN models
}

\section{Krzysztof Katarzyński*t}

Nicolaus Copernicus University, ul. Gagarina 11, PL 87-100 Toruń, Poland

E-mail: kateastro.uni.torun.pl

The new observational capabilities of Cherenkov Telescope Array (CTA) will allow for a more detailed investigation of Very High Energy (VHE) emission of extragalactic sources. We briefly demonstrate what should be possible to observe with this new observatory. We also discuss and point out a few problems that should be solved with this new instrument.

AGN Physics in the CTA Era-AGN2011,

May 16-17, 2011

Toulouse, France

* Speaker.

$\dagger$ 


\section{Introduction}

CTA is dedicated for observations of VHE emission of different types of astronomical object including Active Galactic Nuclei (AGN). This VHE emission, observed from a few tens of GeVs up to hundred TeVs was detected in a few different types of AGNs. Most of those AGNs are blazars. Such sources are radiating usuall in entire range of electromagnetic spectrum, starting from radio frequencies and ending in some cases in $\mathrm{TeV}$ energies. This emission is usually variable in time scales from years up to a few minutes in extreme cases (e.g. Aharonian et al., 2007 [3]). Moreover, in many cases correlation between different energy ranges was found (e.g. Fossati et al., 2008 [5]). It is not clear how such emission is created. However, it is very likely that the radiation comes from particles accelerated inside jets. Therefore, investigation of blazar's activity may give important informations about particle acceleration processes inside astrophysical objects. CTA with sensitivity better by at least one order of magnitude in comparison to currently operating instruments and with significantly better energy thresholds will give new possibilities for such investigation.

\section{What should be possible to observe with CTA}

VHE emission of extragalactic sources, above $\sim 1 \mathrm{TeV}$ can be significantly absorbed. $\mathrm{TeV}$ gamma-ray photons travelling through extragalactic medium may interact with the Extragalactic Background Light (EBL) photons producing electron-positron pairs. This causes the absorption that significantly reduces the number of received $\mathrm{TeV}$ photons and thus is limiting the distance from which we can observe $\mathrm{TeV}$ sources. The significantly better sensitivity of CTA will allow for observations of more distant sources. Therefore, to check more precisely what should be possible to detect with CTA we performed simple calculations. The main assumption for our calculation was that sources similar to objects observed at relatively small redshifts $(z \lesssim 0.5)$ are existing also at larger distances. The methodology of our calculation was the following:

- selected the VHE spectrum of well known source,

- deabsorb the spectrum to obtain the intrinsic emission profile,

- recalculate observed flux and frequency for a larger distance,

- absorb the emission according to the new distance,

- compare the obtained spectrum with sensitivity curves expected for CTA.

To calculate the absorption effect we have chosen the EBL absorption model proposed recently by Kneiske \& Dole, 2010 [10].

First, for our test we selected Mrk $501(z=0.034)$ and the VHE spectrum of this object obtained on 7th of April 1997 by CAT telescope (Djannati et al., 1999 [4]). In April 1997 this object was very active, a few flaring events was observed in this source by many different instruments, the strongest one on 16 of April, when the emission level was almost one order of magnitude higher than on 7th of April. Thus the spectrum selected for the calculation represents rather medium emission level. For most of the time the blazar emission levels are low or medium and this is what 

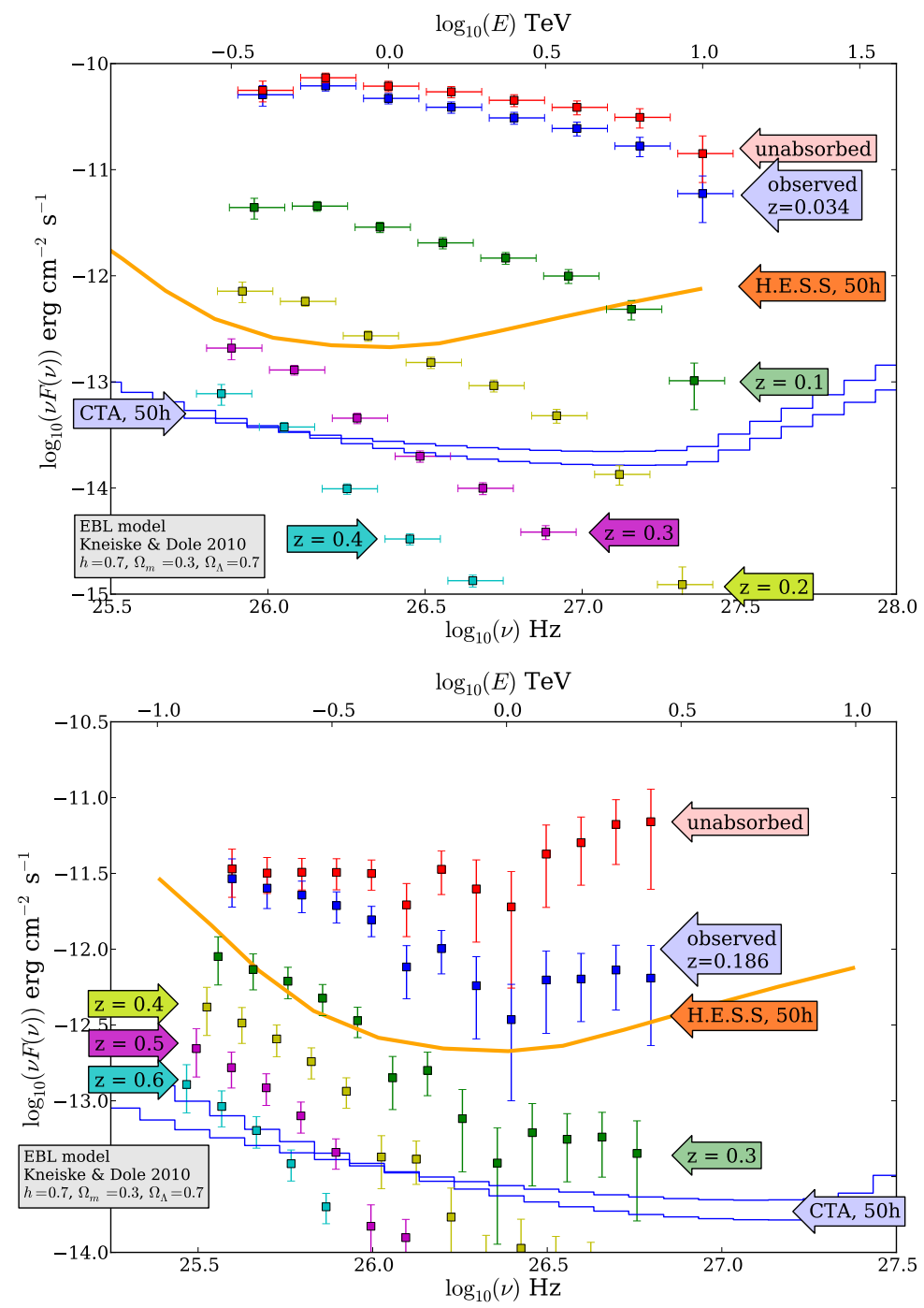

Figure 1: The upper panel shows the spectrum of Mrk 501 observed in 1997 by CAT telescope (DjannatiAtai et al., 1999 [4]) and how this spectrum could look if observed from larger distances. Solid lines show the H.E.S.S. sensitivity curve for 50 hours of integrations and estimated sensitivity of CTA for the same integration time. Lower panel demonstrates emission of 1ES 1101-232 observed by H.E.S.S. experiment (Aharonian et al., 2006 [2]) and also different estimations in comparison to H.E.S.S. and CTA sensitivity curves.

CTA will likely observe. The result of our test is presented in Fig. 1 that shows the possibility to observe sources similar to Mrk 501 from $z \lesssim 0.4$ in energy range from 300 to $500 \mathrm{GeV}$ in 50 hours of integration time.

For the second test we have chosen 1ES 1101-232 $(z=0.186)$, the source with steep intrinsic spectrum and the observations of this object made by the H.E.S.S. observatory (Aharonian et al., 2006 [2]). Such specific slope of the emission gives opportunity to observe the source from significantly larger distances. Indeed, if CTA will achieve the estimated level of the sensitivity it should be possible to observe sources like 1ES 1101-232 at a distance equivalent to $z=0.6$ in a energy 
range of about a few tens of $\mathrm{GeV}$. However, this would require again about 50 hours of observation.

\section{Problems to be solved}

\subsection{Peak correlation}

The spectra of blazars usually contain two characteristic peaks. The first peak comes from synchrotron emission of relativistic electrons and the second peak is created by inverse-Compton (IC) scattering of low energy photons (radio to UV) by high energy electrons. The analysis of the Mrk 421 activity performed by Tanihata et al., 2004 [12] shows an correlation between the position of the synchrotron peak and the emission level at the peak $E_{\text {peak }} \propto v F_{\text {peak }}^{0.77 \pm 0.02}$. In sources that are producing $\mathrm{TeV}$ gamma rays, like Mrk 421, the synchrotron and the IC emission is likely produced by the same population of electrons. Therefore, if the correlation is caused by a specific evolution of the electron energy distribution the same or similar behaviour should be observed in the evolution of the IC peak. However, it is difficult to investigate such correlation because this currently requires simultaneous observation in $\mathrm{GeV}$ and $\mathrm{TeV}$ range by two different instruments. If CTA will cover the energy range from a few tens of $\mathrm{GeVs}$ up to hundred $\mathrm{TeV}$ then is should be possible to investigate this correlation with this single instrument only.

The correlation itself seems to be very interesting from the theoretical point of view. The correlation slope is similar or even identical for rise and decay of flux. This is in contradiction to prediction of many models. In the simplest possible scenario we may assume an injection process that is simulating particle acceleration at a shock front. Such injection should give a relatively fast increment of flux. When the injection stops the radiative cooling should take control over the particle energy evolution and cause a systematic decrease of the emission. The problem is that the injection and the cooling are different physical processes that give different correlation for the rise and the decay of a flare. It is possible to get the same correlation for the rise and the decay of activity if we assume that the activity is caused by a change of the Doppler factor. If the source of VHE emission travels with relativistic velocity and is observed at a small angle (a few degree) then a small modification of this angle due to a curved trajectory should give significant change in the observed flux. Increase or decrease of flux due to the change of the Doppler factor should give the same slope for rise and decay of an outburst. This simple approach can explain how the correlation observed in the synchrotron peak could be created. However, we have no information if there is a similar correlation in the IC peak but it will be possible to investigate issue with CTA.

\subsection{TeV/X-ray correlation}

The simultaneous observations of Mrk 421 made by the Whipple telescope and the RXTE satellite in 2001 (Fossati et al., 2008 [5]) showed an unexpected correlation between $\mathrm{TeV}$ and Xray emission. The correlation can be approximated by a simple power-law function $F_{\mathrm{TeV}} \propto F_{\mathrm{X} \text {-ray }}^{x}$ where the slope of the correlation $x \gtrsim 2$ when it can be measured precisely. The observations of PKS 2155-304 made by the H.E.S.S. observatory and the Chandra satellite (Aharonian et al., 2009 [1]) shows an even steepr slope of the correlation $x \simeq 3$. Such steep slope means much faster change of the IC emission in comparison to the X-ray radiation. It is difficult to explain such fast changes within the standard approach that assumes synchrotron self-Compton scattering inside a 
single source (Katarzyński et al., 2005 [6]). With two or more sources observed simultaneous it is possible to explain any slope of the correlation. However, this requires a special assumption about the relative radiation levels of the $\mathrm{TeV}$ and the X-ray emission of the sources (Katarzyński \& Walczewska 2010 [8]). The best observation of the correlation was obtained for the flare in Mrk 421 on 18/19 March 2001 (Fossati et al., 2008 [5]). This particular activity shows that the correlation slope can be similar or even identical for rise and decay of the flare. This, like in the case of the peak correlation, is problematic for standard models. The models that assume usually an acceleration that causes flux increment and after that the radiative cooling predict two different correlation slopes for rise and decay of activity. It is possible to explain the same correlation for the rise and decay if the activity is caused by the Doppler factor change as it was in the peak correlation case. However, this assumption still requires simultaneous emission of two or more sources to obtain the correct slope (Katarzyński \& Walczewska, 2010 [8]). Therefore, this correlation is quite challenging for standard emission models. The correlation may give significant constraints for the emission models and should be investigated with the maximum possible precision in $\mathrm{X}$-rays and $\mathrm{TeV}$ gamma rays. Unfortunately the precision of currently operating Cherenkov telescopes is significantly worse than the precision of orbital X-ray observatories. CTA will bring us measurement precision comparable to the $\mathrm{X}$-ray precision and therefore will give an unique opportunity to investigate the TeV/X-ray correlation.

\subsection{Hard intrinsic spectra}

The already mentioned spectrum of 1ES 1101-232 is exceptionally hard in TeV energies. This may mean that the emission is produced only by high energy particles $\left(E=\gamma m_{\mathrm{e}} c^{2}, \gamma \gtrsim 10^{5}\right)$. In such a case the intrinsic $\mathrm{TeV}$ spectrum can be as hard as $F \propto v^{1 / 3}$ (Katarzyński et al., 2006 [7]). This particular spectral index comes from the fact that the particles are scattering the "tail" of the synchrotron emission. If the minimum energy of the particles is lower $\left(\gamma \sim 10^{4}\right)$ then the spectrum will be still hard but the spectral index should be significantly smaller. However, it is impossible to obtain accuretly the intrinsic spectrum because we do not know how much the emission is absorbed. According to an old model of the EBL absorption (Kneiske et al., 2004 [9]) the intrinsic spectrum could be as hard as possible $\left(v^{1 / 3}\right)$. However, the new models of the EBL absorption (e.g. Kneiske \& Dole, 2010 [10]) are suggesting that the absorption level is significantly lower and therefore the intrinsic spectrum is significantly softer. To choose which approach is correct it is necessary to conduct observations of 1ES 1101-232 from a few GeVs up to several TeVs. The model that can explain hard spectra is predicting that the emission slope in the $\mathrm{GeV}$ range should be identical to the $\mathrm{TeV}$ one. Moreover, at the energy of about a few $\mathrm{TeV}$ there should be an abrupt break in the spectrum. It is impossible to check those predictions with currently operating instruments but some indirect investigations seems to confirm this theory (e.g. Tavecchio et al., 2009 [13]) However, it should be relatively simple to verify this model with CTA observations.

\subsection{Rapid variability}

On 28 of July 2006 the H.E.S.S. observatory recorded a huge outburst in PKS 2155-304 $(z=0.116)$. In about $1.5 \mathrm{~h}$ the TeV flux increased and decreased a few times by a factor of about 20. That was the fastest ever observed variability in an active galaxy. The variability time scale constrains the size of the emitting region that in this particular case should be about $10^{14} \mathrm{~cm}$. This 
gives a relatively small volume and is suggesting that the source of the emission was very dense or was travelling with a huge relativistic velocity. However, inside a dense source the interactions between $\mathrm{TeV}$ photons and IR synchrotron photons are very efficient, causing also very efficient pair production and then the absorption of the VHE emission. On the other hand a source that travels with velocity equivalent to the Lorentz factor $\Gamma \sim 100$, necessary to explain such rapid variability, is also very unlikely. The radio interferometric observations of central parts of $\mathrm{TeV}$ blazars with angular resolution of about one pc (Piner et al., 2010 [11]) do not show such rapidly moving jet components. Several theoretical solutions were proposed to explain such rapid variability. However, each of them requires some special assumptions about the source parameters or some special "fine tuning". The true nature of such intriguing variability is unknown. It would be interesting to know if such variability occurs only during a flaring state or also at low levels of the emission. For this issue and probably many others that concern rapid variability CTA will greatly improve our knowledge.

\section{Summary}

CTA with new observational capabilities will give an unique opportunity to discovery VHE emission from active galaxies that are at significantly larger distances. We performed simple calculations to demonstrate briefly what should be possible to observe with CTA at higher redshifts. On the other hand there are many problems that concern already known sources. We pointed out a few of them and described how CTA should help. However, probably many other problems will come out with the new quality of the VHE observations that CTA will bring us.

\section{References}

[1] Aharonian, F., Akhperjanian, A. G., Anton, G., et al. 2009, arXiv:0906.2002

[2] Aharonian, F., Akhperjanian, A. G., Bazer-Bachi, A. R., et al. 2006, Nature, 440, 1018

[3] Aharonian, F., Akhperjanian, A. G., Bazer-Bachi, A. R., et al. 2007a, ApJ, 664, 71

[4] Djannati-Atai, A., Piron, F., Barrau, A., et al. 1999, A\&A, 350, 17

[5] Fossati, G., Buckley, J. H., Bond, I. H., et al. 2008, ApJ, 677, 906

[6] Katarzyński, K., Ghisellini, G., Tavecchio, F., et al. 2005, A\&A, 433, 479

[7] Katarzyński, K., Ghisellini1, G., Tavecchio, F., et al. 2006, MNRAS, 368, 52

[8] Katarzyński, K., \& Walczewska, K., 2010, A\&A, 510, 63

[9] Kneiske, T. M., Bretz, T., Mannheim, K., \& Hartmann, D. H., 2004, A\&A, 413, 807

[10] Kneiske, T. M., \& Dole, H. 2010, A\&A, 515, A19

[11] Piner, B. G., Pant, N., \& Edwards, P. G., 2010, ApJ, 723, 1150

[12] Tanihata, Ch., Kataoka, J., Takahashi, T., et al. 2004, ApJ, 601, 759

[13] Tavecchio, F., Ghisellini, G., Ghirlanda, G., et al. 2009, MNRAS, 399, 59 